\title{
THE RECOGNITION AND PREVENTION OF ARTEFACTS OF MARCHI METHOD
}

\author{
BY \\ MARION C. SMITH \\ From the National Hospital for Nervous Diseases, Queen Square, London
}

For more than 80 years now the Marchi method has been used for the demonstration of degenerating nerve fibres. The usefulness of this method is shown by the fact that it has stood the test of time and is still the standard method of demonstrating degenerating myelin. But the method has two main limitations : it is of use only at certain stages of the process of the degeneration and it is marred by the frequent occurrence of artefacts.

In recent papers (Smith, 1951, 1956; Smith, Strich, and Sharp, 1956), my colleagues and I have shown that the Marchi method is far less limited than was previously believed. For it is a useful method of demonstrating degeneration in tracts in man when there is more than a year between the occurrence of the lesion and the death of the patient. And further, it can be used with great effect when the tissue has been stored for many years in formalin.

Since the occurrence of artefacts remains a serious disadvantage of the Marchi method, the major part of this paper will describe various forms of pseudoMarchi staining and indicate how these artefacts can be distinguished from true degeneration. In addition, some technical recommendations will be given ; these are based on considerable experience of the Marchi method in tracing degenerating fibres in the human central nervous system.

\section{THE RECOGNITION OF PSEUDO-MARCHI ARTEFACTS}

The appearance of true degeneration will first be briefly considered, and then the main forms of artefact will be described and illustrated. For descriptive purposes the terms "longitudinal section " and "transverse section" will refer solely to the long axis of the degenerating fibres.

\section{True Degeneration}

The appearance of degenerating fibres in Marchi preparations is related to the period of survival between the occurrence of the lesion and the death $\mathbb{\Phi}$ of the patient. When the period of survival is 20 to 80 days, most, if not all, of the black-stained material derived from the degenerating fibres is extracellular. In transverse section it is in the form $\vec{\omega}$ of round bodies (Figs. 2, 5, and 6). In longitudinalo section it is at first in the form of irregularly swollen $\overline{3}$ threads (Figs. $7 \mathrm{~A}$ and B, Fig. 9) and later as. spherical bodies, which, to some extent, are arranged $\overrightarrow{0}$ in strings (Figs. $8 \mathrm{~A}$ and C, Fig. 10). There $\overrightarrow{\mathrm{d}} \mathrm{e}$ ( also compound granular corpuscles containi $\vec{g} \mathrm{~g} \perp$ numerous black globules lying among the degent-o erating fibres. These increase in number the longerthe survival period. In cases with survival periods? of about 140 days most of the black material has 2 intracellular, and in cases with survival periods $\$$ 250 days or more there is rarely any extracellư black material (Fig. 3). In general, degenerating fibres are more easily recognized in longitudinalo than in transverse section; this applies particularly to fine fibres.

\section{Artefacts}

A constant feature of true degeneration is its $\overrightarrow{\vec{O}}$ continuity : the pattern of staining conforms with the known form of the structures ; the black staining indicates fibres, and thus it may be traced across:a section, from one section to the next and from one block to the next. In most forms of artefact, this feature of continuity is absent; the black staining does not form a pattern consistent with the known structure of nerve fibres. This randomo distribution is the most important criterion by which $₹$ to judge between false and true degeneration.

Some artefacts resemble true degenerating fibres $>$ seen in transverse section, while others resemble degenerating fibres in longitudinal section. They N will be considered in the following three groups, $\mathrm{N}$ of which the first is most important in transverse sections, the second and third in longitudinal sec- $\omega$ tions : (1) pseudo-Marchi dust ; (2) pseudo-Marchio 


\author{
Pseudo-Marchi dust
}

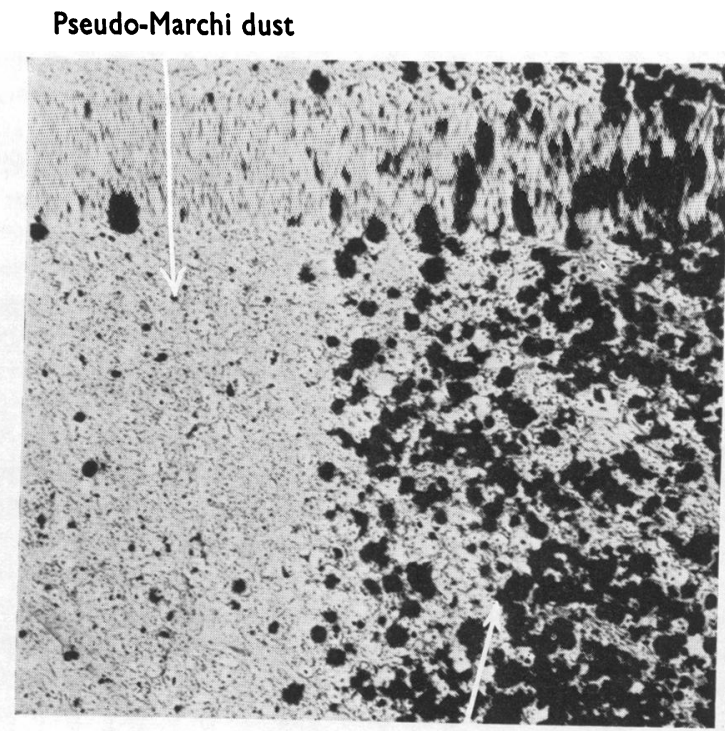

True degeneration of fibres
Fig. I

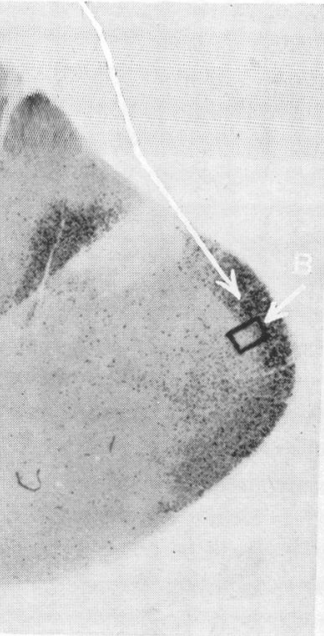

Pseudo-Marchi dust throughout section

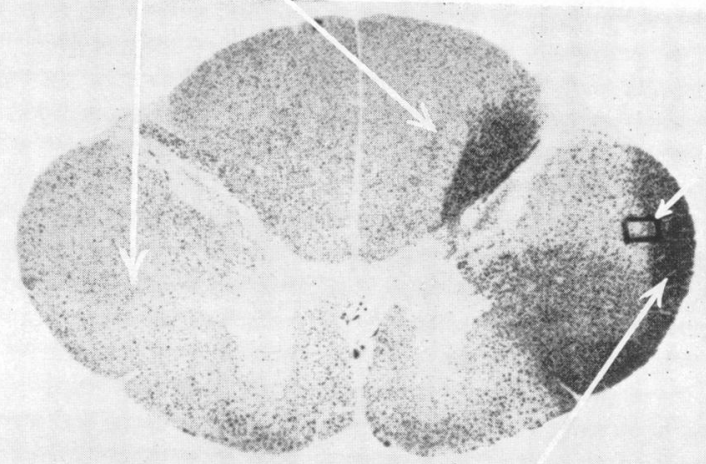

Fig. 2

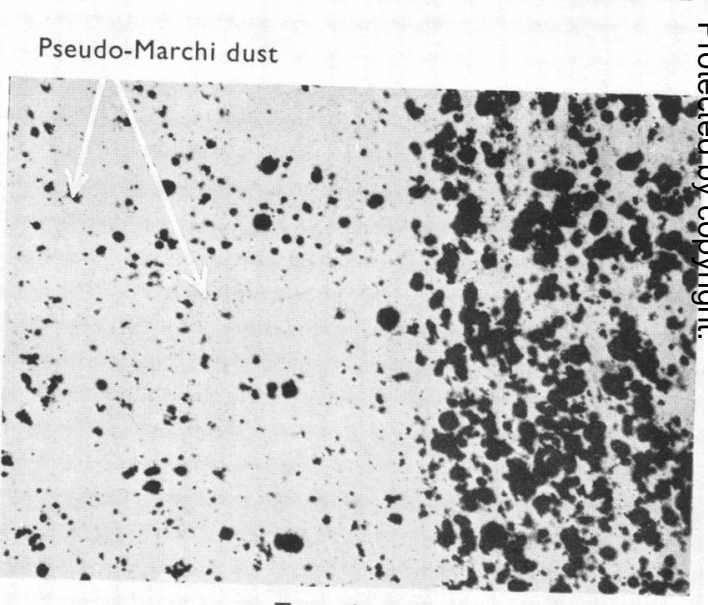

True degeneration of fibres $\mathbf{2 B}$ staining of normal fibres ; (3) massive, localized pseudo-Marchi staining.

The terms " pseudo-Marchi staining ", " Marchi dust", and "Marchi bodies" are used by all workers in this field. Unfortunately there has been no standardization, and so these terms need to be defined by every worker as he uses them. In this paper, the term " Marchi bodies" will be used to indicate true degeneration of fibres. All other terms indicate various forms of artefact.

(1) Pseudo-Marchi Dust.-Pseudo-Marchi dust occurs as fine or coarse particles or as an organized network of particles. The fine and the coarse particles constitute a common form of artefact.
The dust is usually scattered throughout the whole section (Figs. 1 and 2). Sometimes, however, it is more abundant in certain tracts and may even be confined to some tracts (Fig. 4). There is no greater concentration in the area of degenerating fibres than elsewhere. The dust occurs as fine or coarser particles ranging in size from $1 \mu$ up to about $30 \mu$ diameter (Figs. 1, 2, 4, and 5). When this artefact occurs in the form of a fine dust it does not usually cause any difficulties in interpretation; it merely spoils the appearance of the preparation, making it dirty looking. The particles are usually too fine to be confused with Marchi bodies, and, like most other artefacts, they show a random 


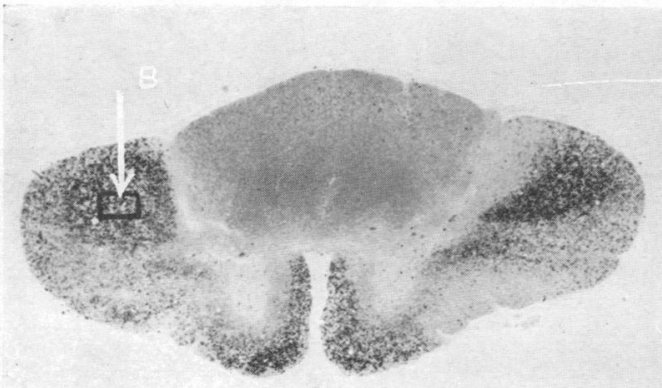

3 A

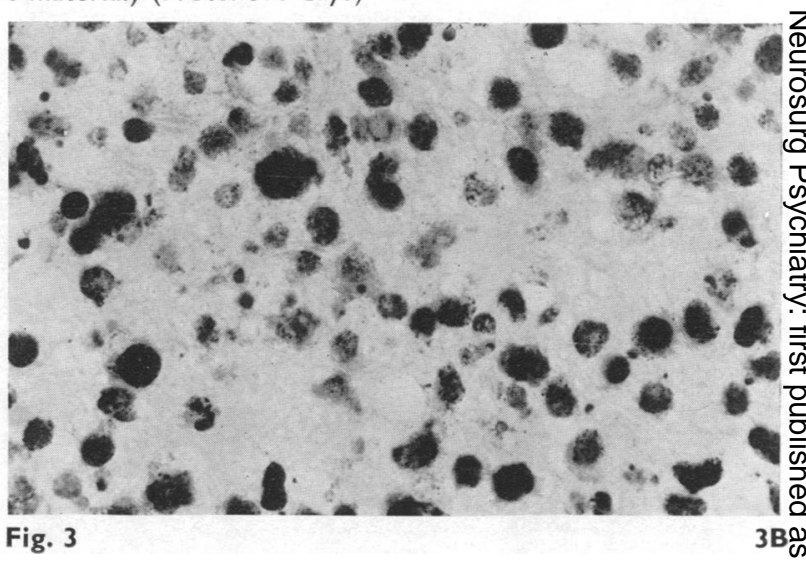

4A

Pseudo-Marchi dust in some normal tracts only
Fig. 3
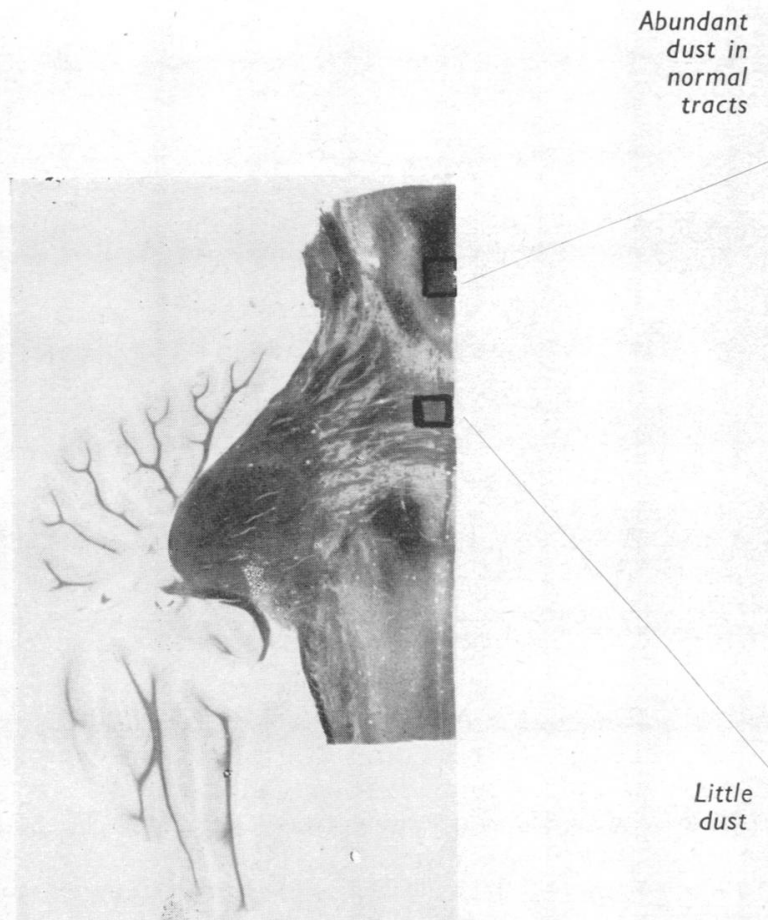

tracts

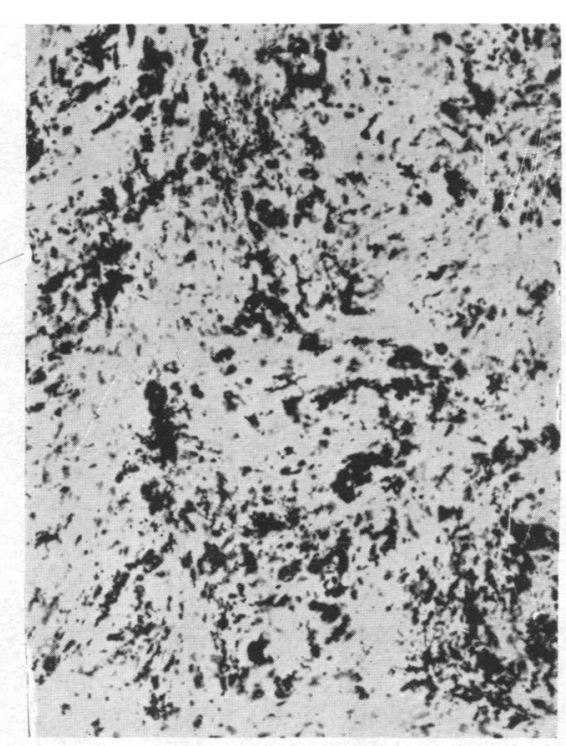

Little

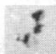

dust

Fig. 4 $\overrightarrow{0}$

$\vec{\omega}$

글

$\overrightarrow{0}$

in

학

융ㅇ

음

물

잉

.

흑ํํ

응

글

ำ

$4 \mathrm{~B} \overrightarrow{\mathrm{O}}$

$e^{2}$

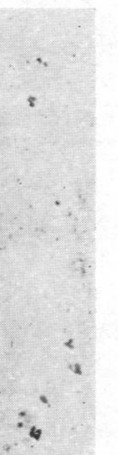

글

웅

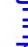

음

3

8

용

믐

N

o

N

श

6

$4 c^{\circ}$ 
distribution. One point of distinction from the rounded Marchi bodies of true degeneration is the angulated outlines of many of the particles (Fig. 2). Many of the larger particles seem to be formed from aggregates of the smaller particles (Figs. 4 and 5). The coarser particles tend to confuse the picture when the true degenerating fibres are all of small diameter, and also to obscure the details on the boundaries of a zone of degeneration, for they may mask the course of a few fibres as the latter leave the main mass of fibres. This is seen in Fig. 2 : there is a peppering of pseudo-Marchi dust throughout the section but the true degenerating fibres can be clearly distinguished. Only on the edge of the main mass do the pseudo-Marchi particles cause confusion. The larger black bodies are only present near the main mass of degenerating fibres, and they can be traced through successive sections. They can therefore be interpreted as degenerating fibres. The fine particles are similar in appearance and number to those scattered throughout the rest of the section, and throughout the whole cord. They cannot be traced from section to section. They are therefore interpreted as pseudo-Marchi dust, although it is possible that a few very fine degenerating fibres may also be present. But there is one further point that makes it unlikely that the fine particles are derived from degenerating fibres : this is the period of survival. In this case the patient lived 81 days after the lesion ; it is uncommon to find evidence of active degeneration in very fine fibres after such a long interval.

The coarse pseudo-Marchi dust particles may sometimes reach such large numbers that they obscure details of the true degenerating fibres, for the whole background of the section is then full of black-stained " bodies". This is shown in Figs. 5 and 6. Each individual black body cannot, in such a section, be infallibly identified as either a degenerating fibre or as pseudo-Marchi dust. But information can still be gained as to the whole pattern of degenerating tracts, which by density and consistency of staining from section to section can be distinguished from the dust, which varies in distribution and amount from section to section.

As was mentioned above, pseudo-Marchi dust may also appear as an organized network. This is a form of artefact which is not easily confused with true Marchi staining, but it may completely hide the true degenerating fibres. The black " particles" form large irregular black masses. A particularly severe example of this artefact is shown in Figs. 6 $B$ and $C$.

(2) Pseudo-Marchi Staining of Normal Fibres. -This form of artefact closely mimics the appear- ance of true degeneration, particularly when seen in longitudinal sections. It tends to occur in certain characteristic sites, such as the larger cranial nerves, especially the second, fifth, seventh, and eighth, and also in the corpus callosum. It seems to be associated with post-mortem damage to the tissues. Fibres which have been pulled on at necropsy become stained over a considerable length; they then resemble fibres which were degenerating before death. They differ from true degenerating fibres in that the diameter of the fibres is constant throughout and in that lengths of the fibre remain unstained while other lengths are stained black (Figs. $8 \mathrm{~A}$ and $\mathrm{C}$; Fig. 11). The true degenerating fibre, it will be remembered, has a beaded or broken-up outline and stains black throughout (Figs. $7 \mathrm{~A}$ and B ; Figs. 8 A and B ; Figs. 9 and 10).

There is, however, another form of pseudo-Marchi staining of normal fibres. If at any time between death and fixation some droplets of myelin escape from the neurilemmal sheath they may stain black with the osmic acid solution (Fig. 11). These stained droplets have the same appearance as Marchi bodies, but can usually be distinguished from true Marchi bodies on account of their distribution : they usually occur in the neighbourhood of fibres showing pseudo-Marchi degeneration and are not particularly associated with the main mass of degenerating fibres which can be traced from section to section. This latter point serves to distinguish them in transverse sections from the true degenerating fibres.

(3) Massive, Localized Pseudo-Marchi Staining. -Occasionally there occurs a very dense patch of black staining, with some characteristics like those described under (2), (Figs. 12 and 13). This massive black staining is particularly liable to occur deep in a large block, in an area of densely packed nerve fibres ; it may enclose an unstained zone. It is characterized by the intensity of the coal black staining, confined to one area of the block. In sections through such an area, the whole region is usually peppered with pseudo-Marchi dust. Every fibre is stained intensely black. Such a mass of intense black staining never occurs with true degenerating fibres. When the fibres are examined, the characteristics mentioned under (2) are seen-the constant diameter and patchy staining of the fibres. In this case the patchiness is demarcated at the edge of the black area, so that all the fibres within the black area are deeply stained, and their extensions beyond it are left unstained. The outlines of some of these stained fibres may be irregular, but the other features serve to distinguish them from degenerating fibres. This form of artefact differs 


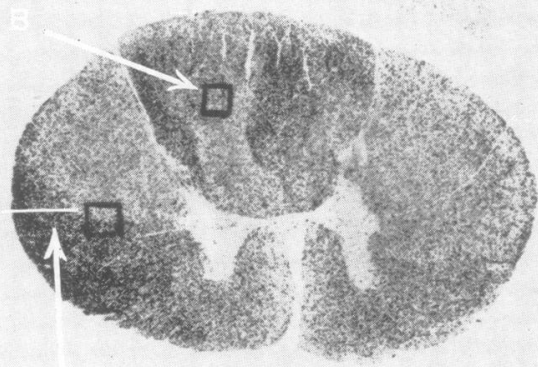

5A True degeneration of fibres (P.O.S. 23 days)

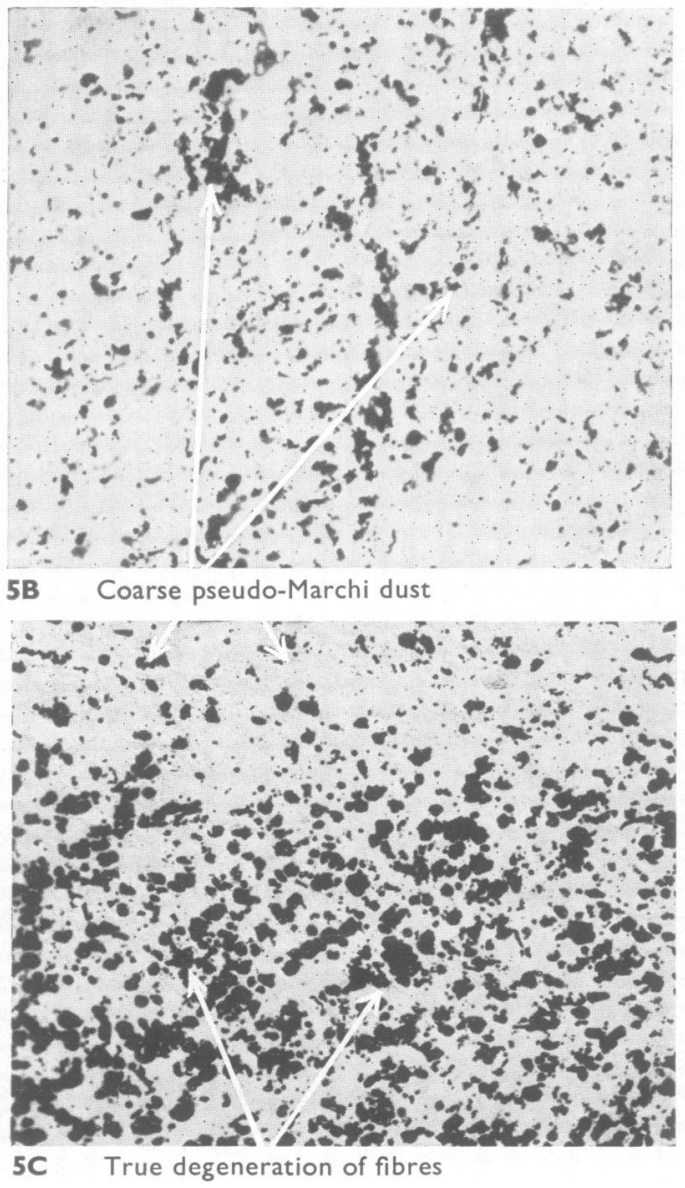

Fig. 5

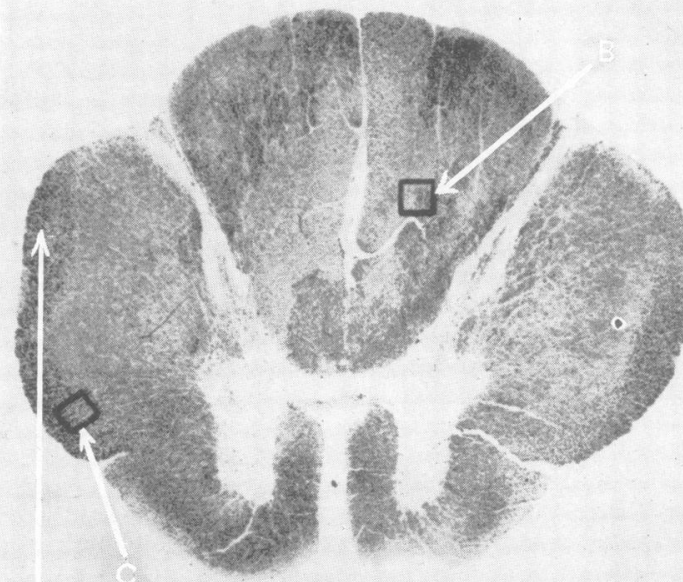

True dageneration of fibres (P.O.S. 75 days)

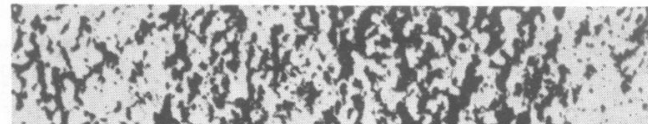

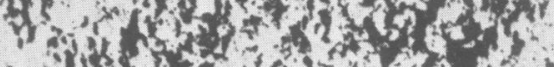

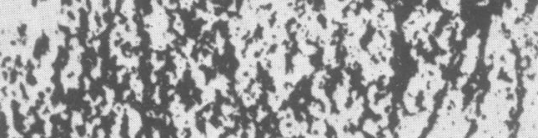

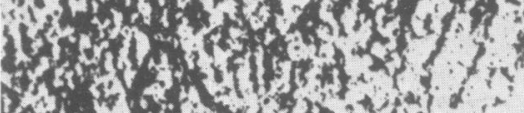

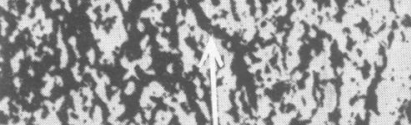

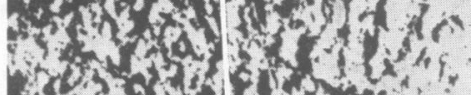

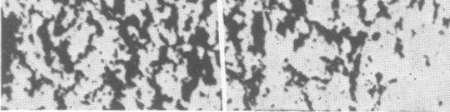

Organized network of pseudo-Marchi dust

6B

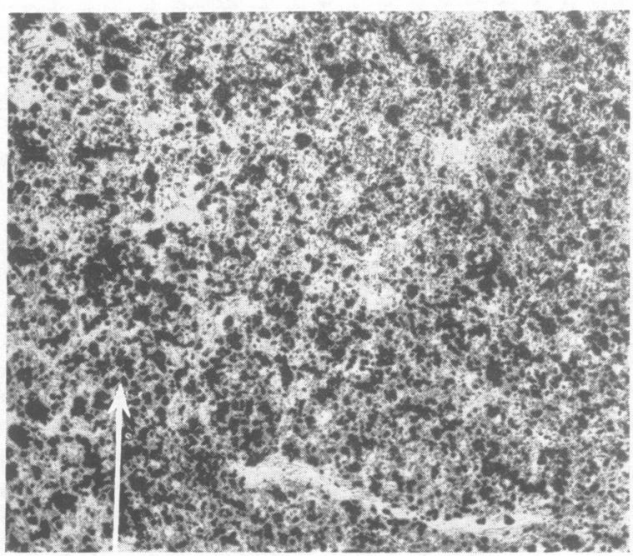

Degenerating fibres hidden by coarse $6 \mathrm{C}$ pseudo-Marchi dust

Fig. 6 
Fine pseudo-Marchi dust

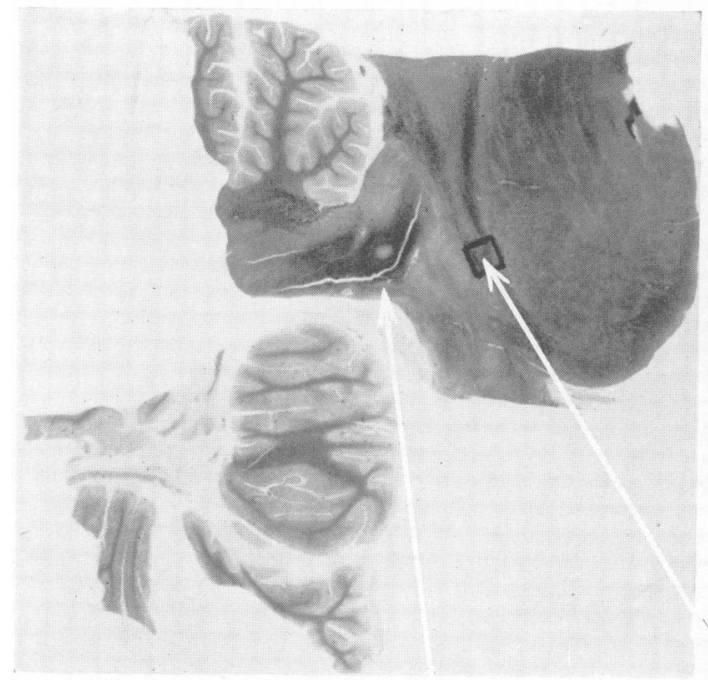

7A Area of non-penetration by osmic acid solution

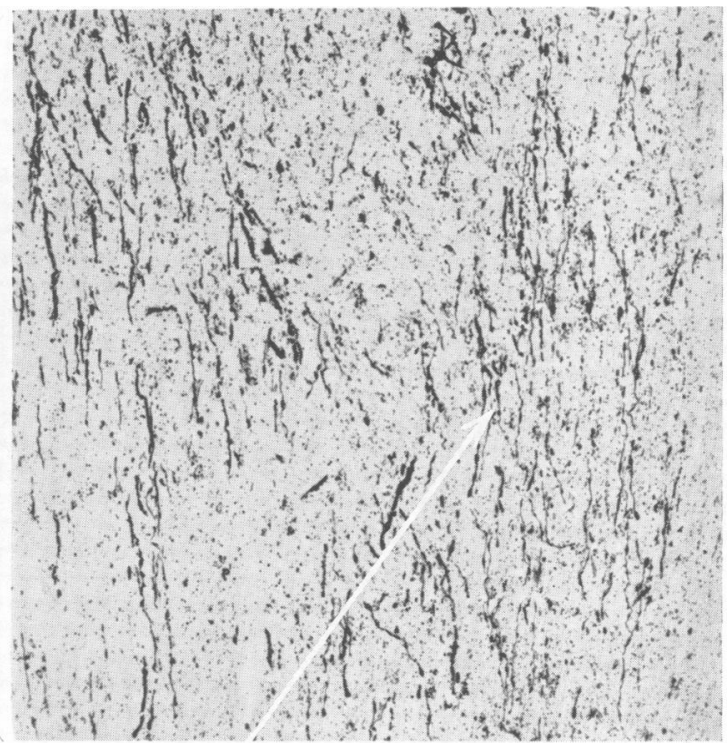

True degeneration of fibres (P.O.S. 23 days)

Fig. 7

Fig. 8

Degenerating fibres (P.O.S. 8 days)

B

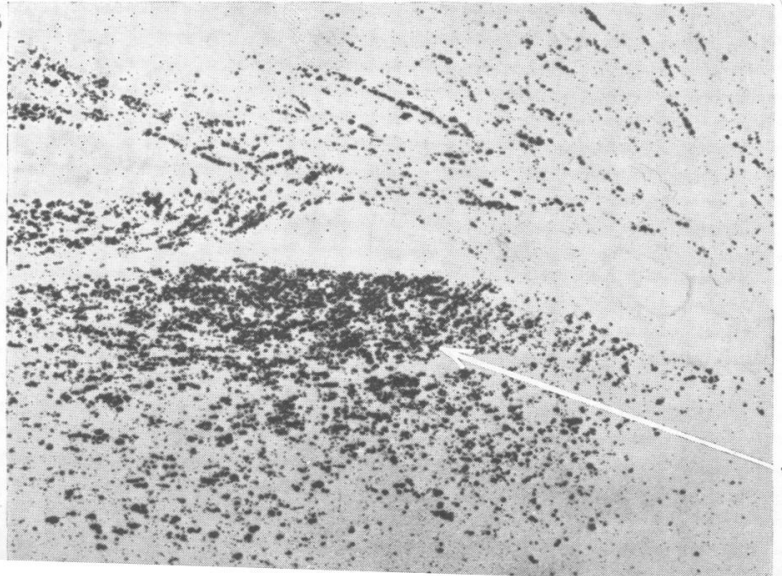

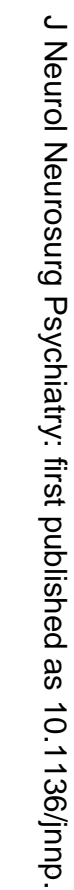

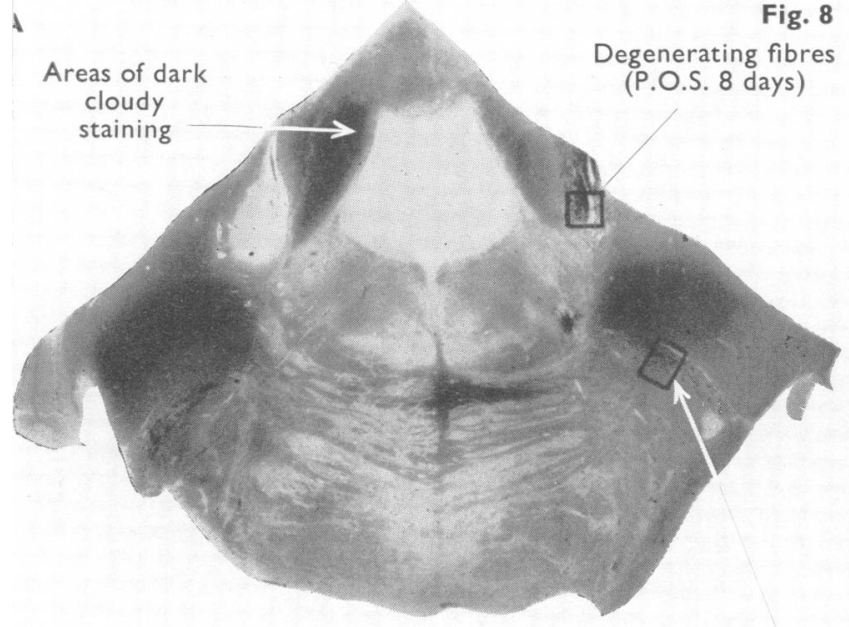




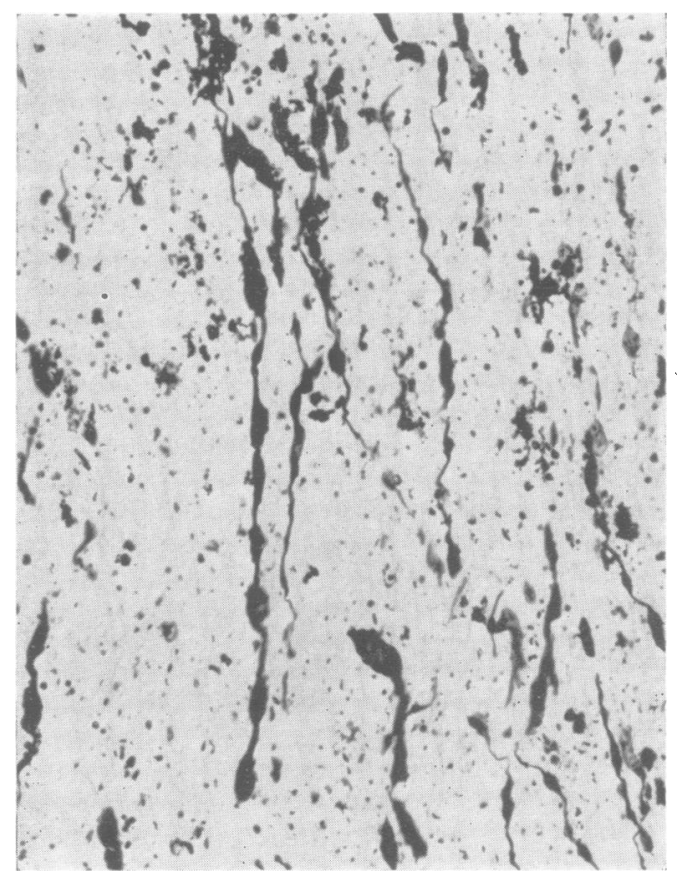

Fig. 9

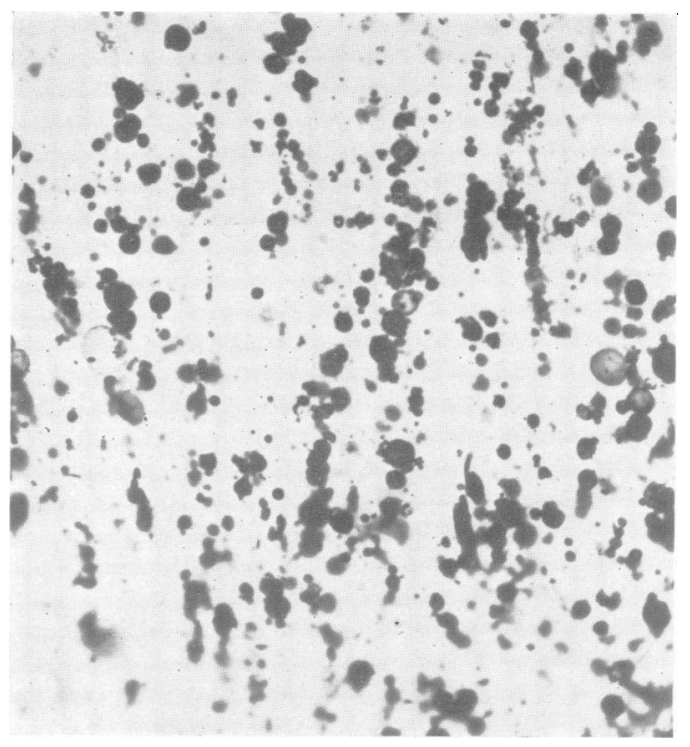

Fig. 10

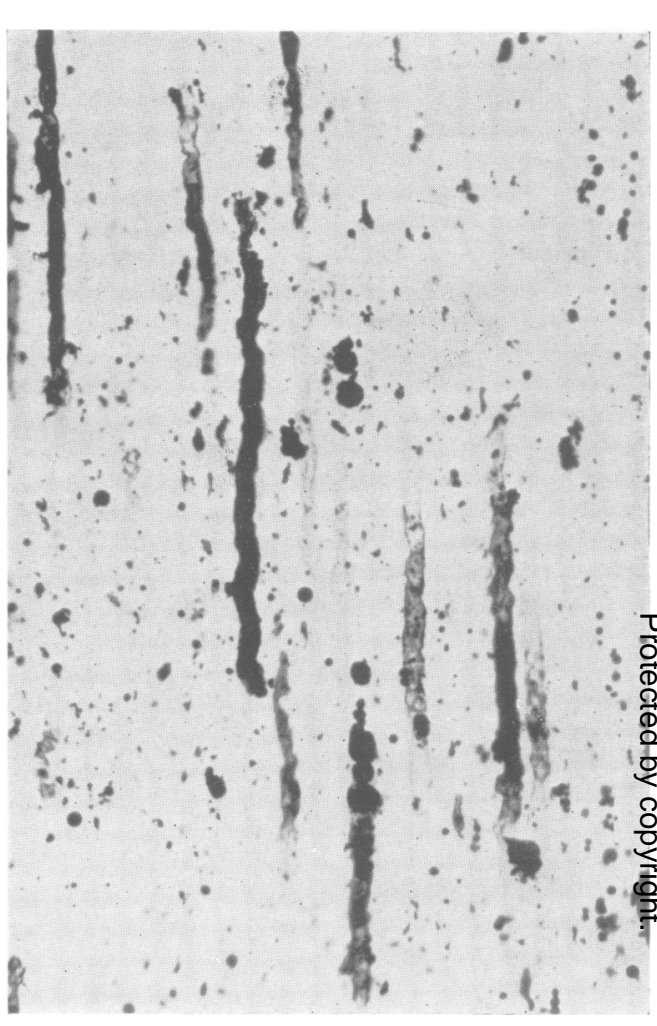

Fig. I I

Fig. 9.-True degeneration of fibres (P.O.S. 23 days) Fig. 10.-True degeneration of fibres (P.O.S. 81 days) Fig. II.-Pseudo-Marchi staining of normal fibres. P.O.S. = reriod of survival 
Massive pseudo-Marchi staining

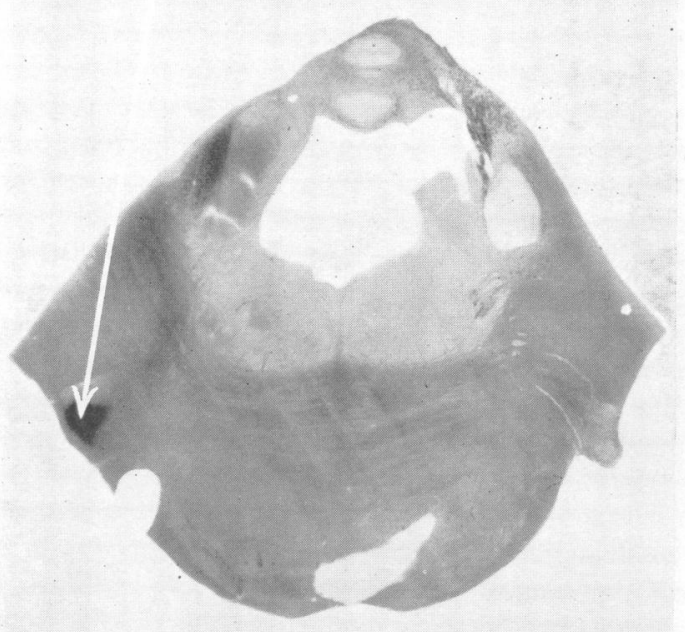

A

Massive pseudo-Marchi staining surrounding area of non-penetration

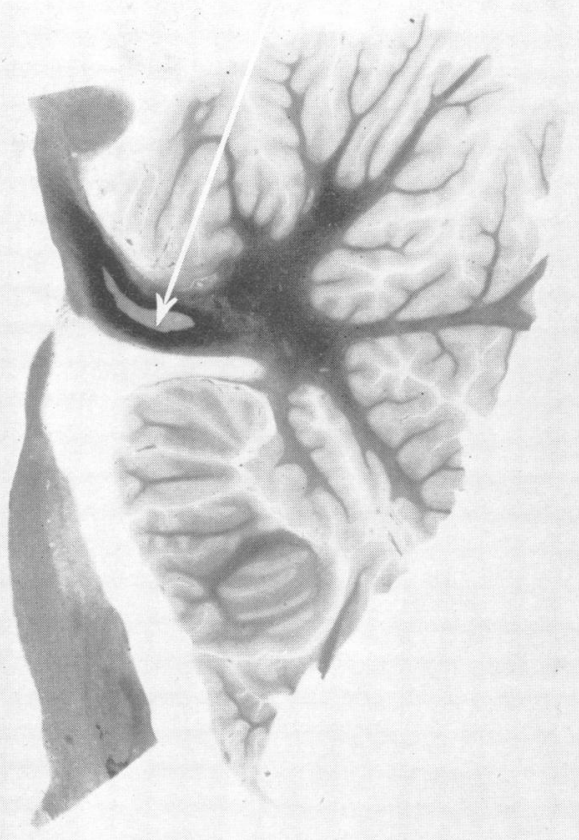

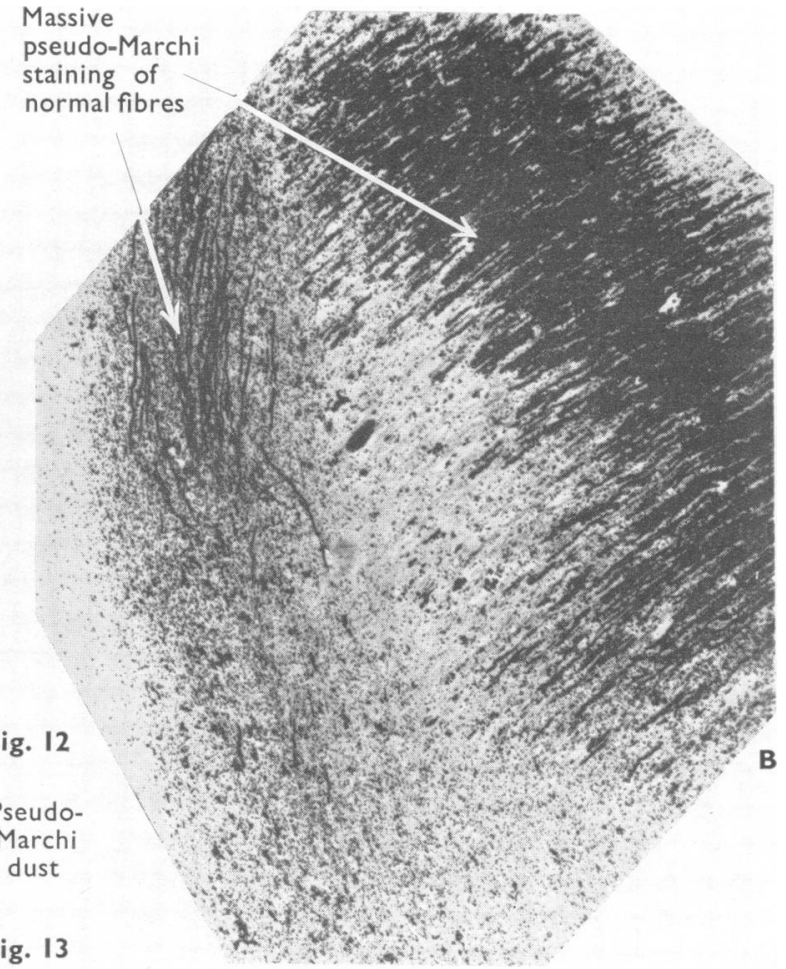

Non-penetration of osmic acid fluid

Intense localized staining of fibres

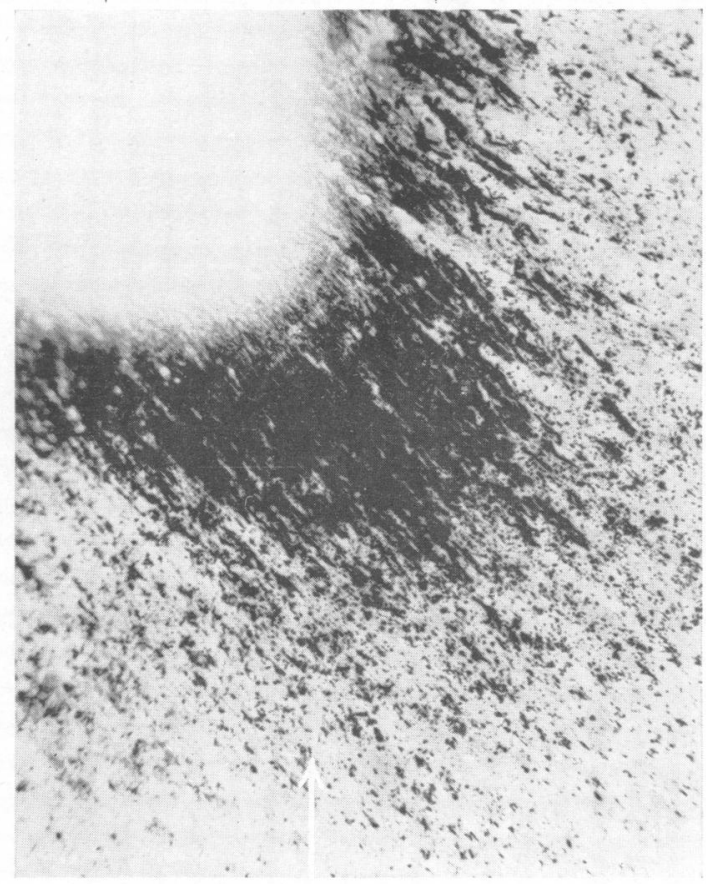

Pseudo-Marchi dust

B

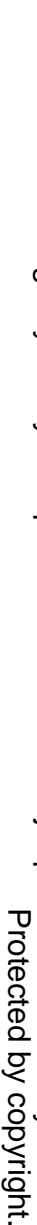


from true degeneration in that the black staining does not spread along tracts or bands of nerve fibres and it is not found in neighbouring sections once the edge of the patch has been reached.

One further feature of Marchi preparations is the occurrence of dark cloudy areas, resulting from irregular depth of staining (Fig. 8). With high magnification, such an area is more deeply stained than the rest of the section, and is brown not black. The appearance in no way resembles degeneration of fibres.

\section{THE PREVENTION OF ARTEFACTS}

As was stated above, no method has been discovered by which artefacts can be entirely prevented. There is already a large literature devoted to ways and means of avoiding them. I have found the following papers particularly useful : Duncan $(1930,1931)$, the latter including a comprehensive review of the literature up to that time; Mettler (1932); Swank and Davenport (1934, 1935); Weisschedel and Jung (1939) ; Mettler and Hanada (1942). The method of impregnation used on most of the present material is essentially that of Swank-Davenport-Glees (Glees, 1943).

The recommendations given below, unless otherwise stated, are applicable to the Swank-Davenport-Glees method, and also, as far as my experience goes, to other versions of the Marchi technique.

\section{Gentle Handling of the Tissue}

At all stages from necropsy onwards extremely careful and minimal handling of the tissue is essential. Even slight tension on nerves must be avoided. In my experience it is immaterial whether the brain and spinal cord are removed in the fixed or the unfixed state : equally good preparations are obtained. It is the gentleness of handling the specimens which is important. At every stage the blocks must be prevented from drying.

\section{Fixation}

The length of the period of fixation does not appear to have much influence on the occurrence of artefacts. Contrary to general belief, in tissue stored in formalin for many years artefacts have been consistently absent.

\section{Choice of Size of Blocks}

One of the drawbacks of the Marchi method is that the osmic acid solution tends not to penetrate the blocks. In some instances the area which is not impregnated is surrounded by dense artefact formation. Owing to this lack of penetration workers have tended to use small and thin blocks. But when the blocks are too thin there is another difficulty : the tissue is brittle and is inclined to warp. In my experience the blocks must be thin, but they need not be small. Three $\mathrm{mm}$. has proved the best thickness for large blocks, and $5 \mathrm{~mm}$. the best for small blocks.
When selecting blocks of tissue it is important decide whether it is more desirable to secure an uninte rupted picture of a degenerated fibre and its relations or to secure a block in which there is definite impregnation at all levels. In some instances it is better to risk now penetration deep in the block, and to obtain a largeg section showing all the ramifications of degenerating nerves, than to cut serial sections throughout the bloc용. It should be noted here that non-penetration is not inevitable in large blocks, but the chance of its occurrente is greater. I have obtained good results with block measuring $90 \times 60 \times 3 \mathrm{~mm}$.

Precautions during Impregnation with Osmic Acid

When making up the osmic acid solution, as soon the capsule of the osmic acid crystals is broken, the crystals must immediately be put into the water and the bottle corked. The reason for this is the rapidity of loss of strength of the acid by vaporizing.

Adequate impregnating solution for the number of blocks is essential, e.g., about $200 \mathrm{ml}$. to one large block. or 10 small blocks (e.g., transverse blocks from the spinal cord). With large blocks, particular attentio has to be paid to ensure that they lie on a flat surface at all stages of preparation. It is essential to averi superimposition of blocks when in the osmic ecrid solution. With all blocks better penetration is achiedy if they are turned over every two days, so that gacte side is equally exposed to the solution. This methot has been found more satisfactory than suspending ghe blocks of tissue as some workers have done ; this latteg method was found to be suitable only when theregire a few blocks and when these are small.

As noted by other workers the following precautsis have been found to be of value : the tissue, when in the impregnating solution, should be kept in the darkg tight-fitting non-metal tops should be used for the containers of fluid and tissue, in order to avoid an escape of osmic acid vapour, or reaction with the meta

\section{Treatment after Osmic Acid Solution}

As recommended by Swank and Davenport, cop tinuous washing in running water for 24 to 48 hous after impregnation is essential. If celloidin or paraffif preparations are being made, the time taken over each stage of dehydration, clearing, and embedding of the blocks should be the shortest possible.

\section{Advantage of Frozen Preparations}

A most important aspect of artefact formation its absence or infrequence in frozen preparations. The appearance of such sections, compared with adjacenf celloidin sections, suggests that dehydration is a frequern factor in the production of pseudo-Marchi artefacts. The following investigation was made to test this. Frozen. sections were cut from blocks after treatment with the impregnating fluid, and the block was then dehydrater and embedded in celloidin as usual. The frozen anfP celloidin sections were then compared. In a number of instances numerous pseudo-Marchi artefacts were foung in the celloidin sections, while the frozen sections were 
perfectly free from them. For this reason frozen preparations may be recommended for all work utilizing the Marchi method if serial sections of the blocks are not required. When complete series of sections are essential, celloidin preparations are easier to handle.

This brief account of measures found useful in limiting the production of artefacts in Marchi preparations is not a theoretical review of the method, but it is intended to be of some practical value to those inexperienced with the method.

\section{Summary}

The Marchi technique remains the best method of demonstrating degenerating nerve fibres within certain limits of time. Recent work has shown that these limits can be extended. The occurrence of artefacts remains the most serious disadvantage of the method.

Illustrations and descriptions of the various forms of artefacts are given, and ways of distinguishing them from true degeneration of nerve fibres are presented.

Some practical precautions in preventing the formation of artefacts are recommended.

I should like to thank Dr. E. A. Carmichael for his encouragement and advice, Mrs. J. A. Mills and Mr. Peter Sharp for all their careful technical work, and Mr. J. A. Mills for his generous help in taking the photographs.

\section{REFERENCES}

Duncan, D. (1930). J. comp. Neurol., 51, 197.

(1931). Arch. Neurol. Psychiat. (Chicago), 25, 327.

Glees, P. (1943). Brain, 66, 229.

Mettler, F. A. (1932). Stain Tech., 7, 95

- and Hanada, R. E. (1942). Ibid., 17, 111.

Smith, M. C. (195i). Journal of Neurology, Neurosurgery and Psychiatry, 14, 222 1956). Ibid. 19,67.

Strich, S. J., and Sharp, P. (1956). Ibid., 19, 62.

Swank, R. L., and Davenport, H. A. (1934). Stain Tech., 9, 11, 129. Wwan, (1935). Ibid., 10, 45, 87.

Weisschedel, E., and Jung, R. (1939i. Z. Anat. Entw Gesch., 109, 374. 